\title{
How to PROceed? Reviewing obstacles and perspectives in patient-centered digital care in radiation oncology
}

\author{
Alexander M.C. Böhner ${ }^{1,2 *}$, Leonard Christopher Schmeel ${ }^{1 *}$, Andreas Feisst ${ }^{1}$, Davide Scafa ${ }^{1}$, \\ Gustavo R. Sarria ${ }^{1}$, Adriana Torres Crigna ${ }^{1}$, David Koch ${ }^{1}$, Stephan Garbe', Barbara Link ${ }^{1}$, \\ Dilini Brüser ${ }^{1}$, Mümtaz A. Köksal ${ }^{1}$, Christoph Henkenberens ${ }^{1}$ and Frank A. Giordano ${ }^{1}$ \\ 1 Department of Radiation Oncology, University Hospital Bonn, University of Bonn, Venusberg Campus 1, 53127 Bonn, Germany. \\ 2 Institute for Experimental Immunology, University Hospital Bonn, University of Bonn, Venusberg Campus 1, 53127 Bonn, Ger- \\ many. \\ * Authors contributed equally
}

\begin{abstract}
As the general public is increasing their online presence and is becoming confident with the digital infrastructure, an opportunity for patient-centered digital care has arisen. Electronic patient-reported outcomes, (e)PRO in short, may facilitate enhanced clinical management of radiation oncology patients. This might enable the physicians to take the initiative and counteract symptoms or undesired side effects before they aggravate and thus, reducing treatment-associated costs. In this article, we review the impetus for and modalities of (e)PRO-based data acquisition and handling in research and routine. We conclude that prospective and technical studies are needed to prove the clinical significance of (e)PROs to pave the way to monetary compensation and widespread application.
\end{abstract}

Keywords: Clinical guideline, cancer treatment, machine-readable documents, markup language, oncology informatics

Oncology is one of the most rapidly advancing disciplines in medicine. Evidently, groundbreaking progress has been made in the past two decades [1-6]. Therapeutic strategies are becoming increasingly complex and involve a broad variety of individual medical specialists $[7,8]$. Inherent to the great opportunities of nowadays, oncology lays several risks that threaten both the success of treatment and patients' wellbeing such as missing hints of undesired side effects in early stages. In some cases, severe symptoms triggered by radiotherapy force a break in treatment, thereby limiting its effectiveness [9]. This provides an impetus to constantly improve clinical case management $[10,11]$, particularly during an ongoing pandemic [12-14]. In current clinical practice, the absence of digital care hampers the physician's ability to be 'in touch' with the patient and act to ensure smooth application of treatment. With digital care implemented, information could be obtained before, during and after therapy to foster our understanding of the impact of a disease - and the treatment - on the patients. A more profound knowledge may support the clinical decision-making process, such as in the selection of a therapeutic approach that yields a more favorable set of possible side effects, as prioritized by the individual patient [15]. Beyond the improvements for the patients, optimized clinical case management might be more economical, both regarding financial expenditures as well as the necessary time to execute actual treatment. High hopes are raised in healthcare providers to eventually dedicate more time to personal interaction with the patient.

Traditionally, the patient-physician interactions are 'turnbased' (Figure 1) [10]. Typically, during treatment, periodic appointments are regularly scheduled, in which physicians interview patients regarding symptoms and other possible therapy-associated problems. As patients vary in preparedness or ability, those meetings mostly do not convey the complete situation to the physician. Patients may also prioritize symptoms and problems differently than the physician would $[10,16]$. However, the latter has to - based on prior documentation and subjective impressions from the interview - evaluate the imminent response to the existing symptoms and decide whether the oncological treatment, supportive therapy or medication has to be adjusted. In conjunction, a risk of missing options for opportune and accurate management arises, postponing this for future visits [17]. For other interventions that involve healthcare providers with different specializations, the elapsed time-to-intervention and time-to-information for the oncologist in charge may be even longer. As the intervention is issued several days or weeks before it is actually conducted, the patient's status may alter in the meantime. Thus, this relevant knowledge may not be accessible by the interventionalist or even the primary oncologist. In summary, anticipating adverse effects and acting accordingly demand that the physician has vast

This article is licensed under the Creative Commons Attribution 4.0 International License. To view a copy of this license, visit http://creativecommons.org/licenses/by/4.0/ or send a letter to Creative Commons, PO Box 1866, Mountain View, CA 94042, USA. Creative Commons Attribution 4.0 International license. (oc) EY 


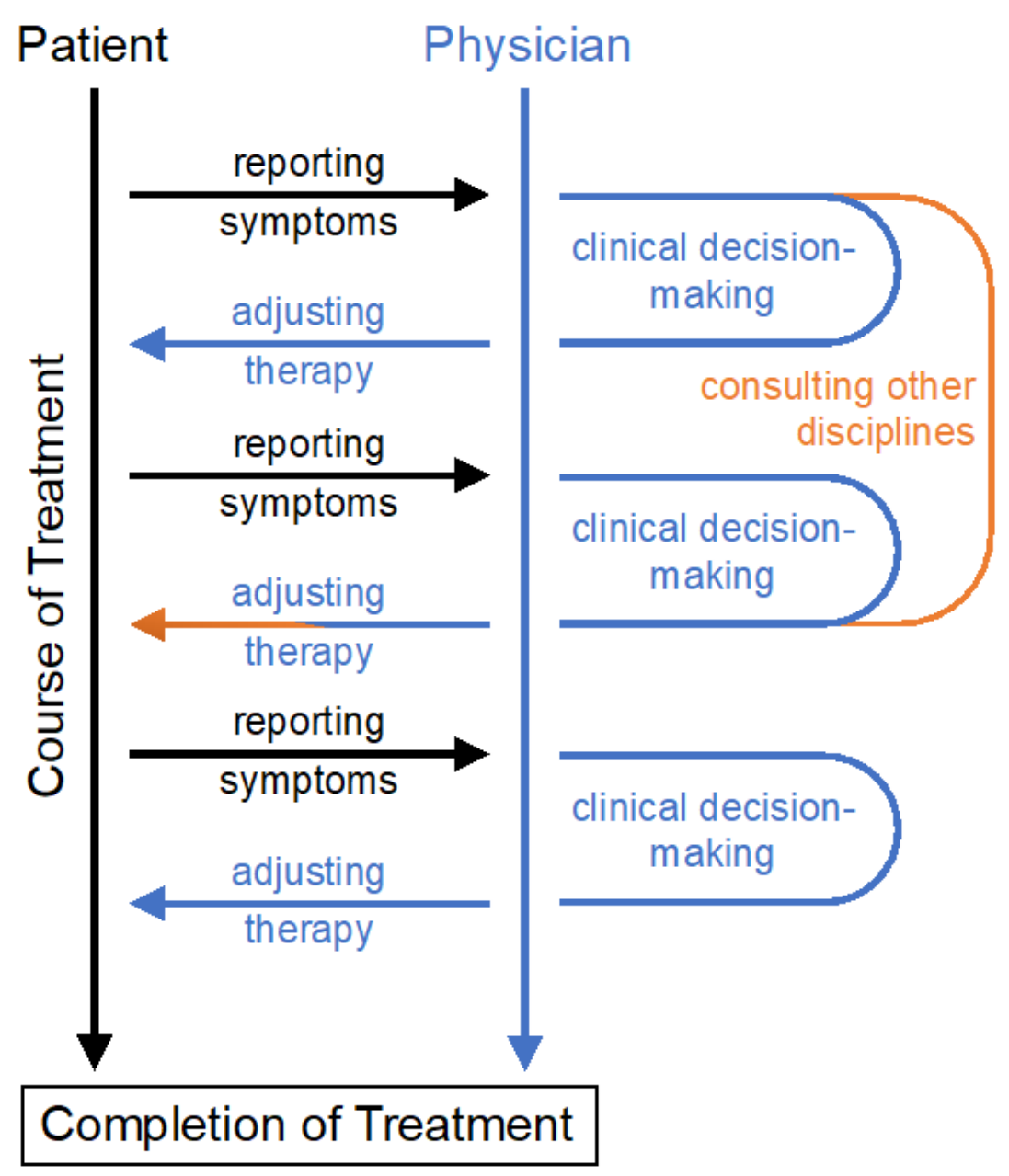

Figure 1. Traditional clinical procedures.

clinical experience, and the process is often based on probability [18].

Through the advent of smartphones and connection to the internet in most of the developed countries, new strategies for clinical case management have become a reality. In the USA, for example, ca. $85.8 \%$ of the population has access to digital infrastructure [19]. Dedicated healthcare apps are already functional and tasked collecting information and channeling the filtered data directly to the healthcare provider, resulting in a more comprehensive and complete assessment of the patients' status $[20,21]$. Thus, the patient is able to answer single-item questionnaires in a setting most comfortable. Moreover, these (electronic) patient-reported outcomes ((e)PRO) would be more elaborate and precise as the patient has adequate time and a low-stress environment to develop the answers while also reducing the possibility of false perception in a personal interview. (e)PROs can be subdivided into two complementary categories: Assessment tools for health-related quality-of-life (HRQL) are exclusively observatory for academic research; items concerning the adverse event monitoring (AEM) are included in the questionnaires in order to alert and trigger an action by the healthcare provider [22]. Both taken together, treatment progression, the kinetics of adverse effects, their counteractions, and the impact on the overall quality of life can be recorded in a straightforward manner. Investigations of (e)PRO as well as HRQL data will spur clinical research and may aid patients and physicians to decide on a certain treatment strategy based on the patient's individual aspects of well-being (if distinct strategies do not vary in efficacy and cost) [15]. Measuring treatment success by HRQL is already the case for a variety of medical disciplines [23-26]. For the particular case of radiation oncology, where patients could develop a late onset of secondary effects, this approach results are of relevance as they help in bridging the patient-physician communication gaps.

In the concrete example of (e)PRO-based clinical care presented in Figure 2, a patient is clinically monitored by the 


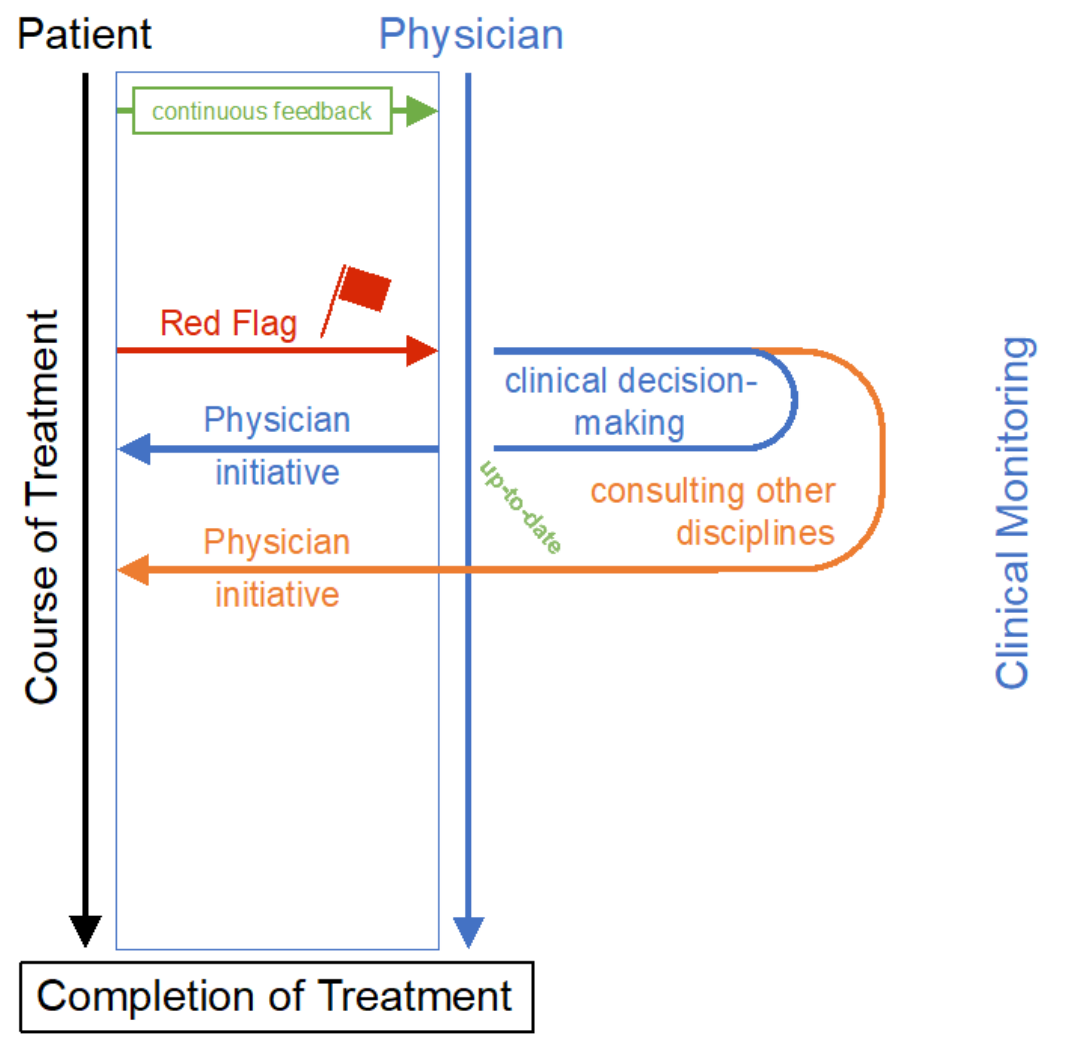

Figure 2. Digital (e)PRO-based clinical procedures.

primary oncologist throughout the complete course of treatment. When predefined values or dynamic parameters related to AEM are exceeded, a 'red flag' is signaled. Notification thresholds may be set at a low level, anticipating the aggravation of adverse effects. In turn, this allows the primary oncologist to take the initiative and, ideally, counteract and ameliorate the adverse event in an early stage via direct intervention and/or via consulting other medical specialists. The latter might be granted access to the (e)PRO data, which would enable the interventionalist to be up-to-date when the indicated treatment is initiated.

Although the opportunities provided by the implementation of (e)PROs into clinical routine seem undoubtedly appealing, certain pitfalls must be avoided to orderly introduce (e)PRO-based clinical decision-making and data collection. On one hand, the (e)PRO tools and apps must be designed for maximum practicability for the patient and the physician alike. On the other hand, clinical studies must be reconceptualized to allow for proper scientific validation.

\section{Striking the right balance - obstacles to proper decision making}

As (e)PRO tools increasingly become implemented into clinical routine, patients and physicians must conform to these new procedures - and establish a certain etiquette to follow. These steps are necessary to maintain the patients' adherence to therapy and (e)PRO data obtainment. Equally important is filtering and prioritizing of the incoming results for the physician to avoid neglecting relevant developments or to overwhelm the healthcare provider hampering the ability for adequate clinical decision-making. This raises the question: What should a 'red flag' alert actually be? The answers to this quintessential question are different from one patient or a cohort of patients to another, although it should be noted that several concerns must be taken into consideration: Patients hold different opinions regarding (e)PRO reporting, which might allow for patient categorization and, subsequently, an automated definition of case-specific notification thresholds. For example, a sudden lack of participation by a usually highly disciplined patient may indicate a clinical problem, whereas an equal lack of commitment by a patient displaying low adherence to (e)PRO data collection should not prompt an equal notification to the healthcare provider. If the notification thresholds are not adjusted to the individual case, the totality of the presented data may cloud the physician's view of relevant pieces of information. Furthermore, patients with unmentioned resentment of (e)PRO procedures may feel 'rejected', and some may even be animated to try to 'deceive' the system by either ceasing to report or barraging the system with irrelevant reports or messages, falsely indicating health deterioration. Both strategies 


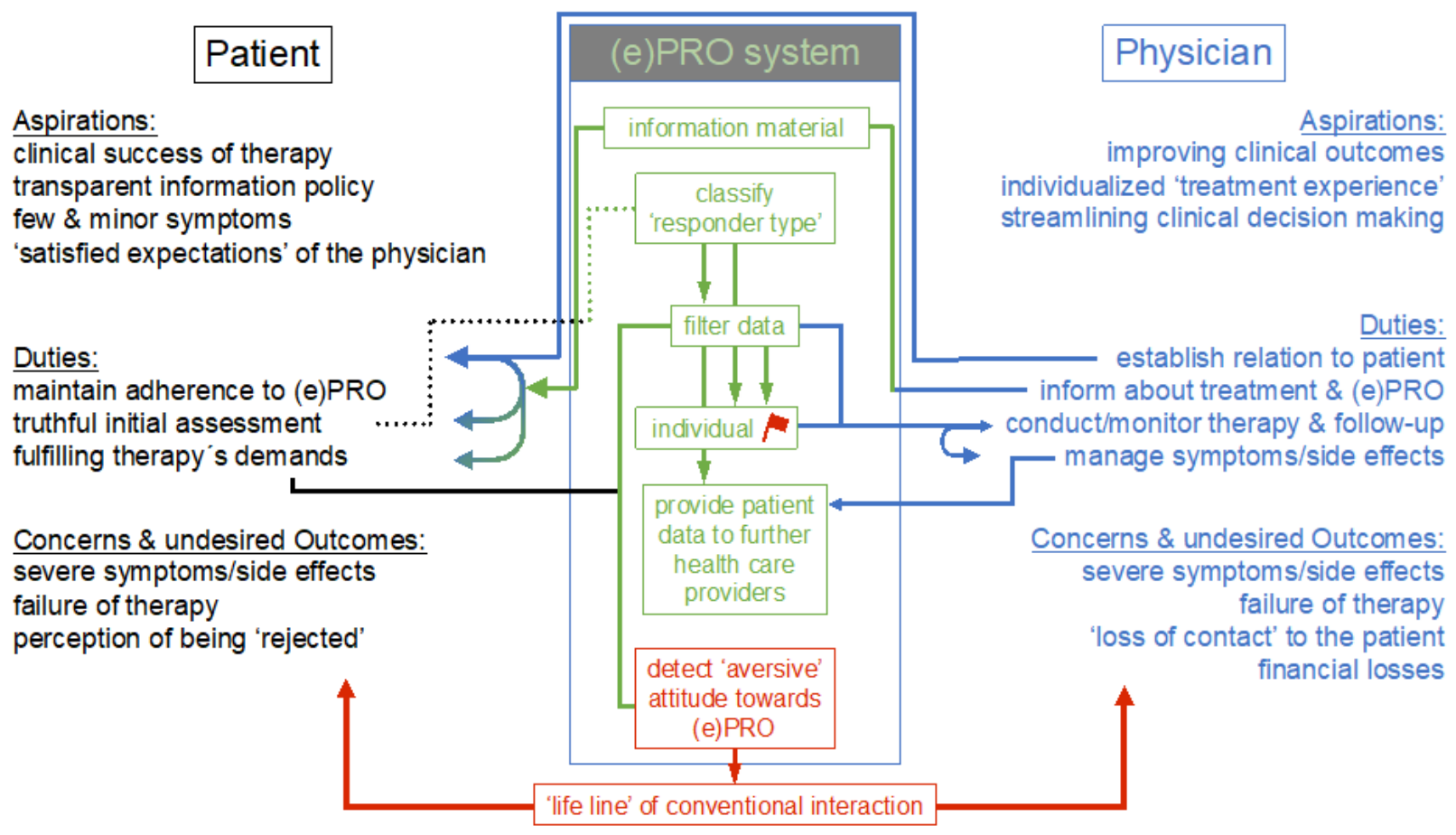

Figure 3. Schematic procedures for (e)PRO deployment.

are aimed at prompting a 'human' response; however, these strategies might be foreseen by automated mathematical analyses at the initial encounter of the patient. Nevertheless, once a patient's attitude towards (e)PRO data collection and usage is suspected to be aversive, traditional clinical routines must automatically take scene to facilitate the continuation of therapy. Prerequisitive to the implantation of any (e)PRObased clinical decision-making, the patient must be assured of the opportunity to see a physician in person regardless of coherence to digitally aided care.

Proving clinical significance - a long and winding road ahead

The implementation of clinical routines depends heavily on the practicability and the monetary compensation for the implementing healthcare provider. In European healthcare systems, financial compensation for clinical procedures generally requires a prior proof of effectiveness. Studies elaborating these clinical advantages by utilizing (e)PRO-based patient-physician interaction systems must address numerous novel pitfalls in study design, as abovementioned. In consequence of these aspects, the real-life applicability of nowadays` studies must be carefully reviewed.

Several studies have investigated the clinical significance of (e)PROs in terms of practicability within the clinical routine for a variety of medical disciplines [22,27-31]. An increasing number of studies utilizing (e)PRO data have shed light on scientific and medical questions in the field of radi- ation oncology $[27,30,31]$. In one study, the rate of questionnaire completion was in excess of $85 \%$ for most measures [27]. Digitalization and use of modern-day digital infrastructure may further elevate patient participation. For most patients, the intent to participate can be presumed, even if some items of the questionnaire would address delicate questions such as sexual function and urine continence [22,27]. The extensive studies on the obtainment and proper management of (e)PRO based clinical routine have paved the way for its wide spread application and have generated an impetus for monetarization by the healthcare provider or healthcare system [32,33]. (e)PRO data was applied not only with the intention of enhancing therapy, but also to assess the effectiveness of (e)PRO data acquisition, for example in the evaluation of swallowing function in particular for patients undergoing head neck cancer radiotherapy [32]. Surprisingly, the investigators could extrapolate that the subjective perception of symptoms declined over time, whereas the physiological performance worsened. This provided a new insight for the aims of patient-centered radiation therapy. A recent publication elaborated on the clinical significance of (e)PRO data and identified promising items in the questionnaires [33]. They also concluded that to monitor the analyzed cohort of head and neck cancer patients, a questionnaire with fewer than the 27 items tested was suitable in clinical routine. Henceforth, prospective studies on the field of oncology, and particularly radiation oncology, will have to be executed in order to compare the effectiveness of (e)PRO-based patientphysician interactions and clinical management to that of conventional procedures. 


\section{Concluding remarks and perspectives}

Broad clinical implementation of (e)PRO-based data acquisition and clinical decision making has proven to be a promising but difficult task. Several studies have assessed a variety of applications and technical platforms, which can improve a patient-centered healthcare and may even positively influence the survival, but are not yet the standard approach. To this end, smartphones in combination with desktop computers might be most appealing to the public, as society increases their online presence [19]. An analog questionnaire - with further digital processing at the healthcare center - might reach out to those lacking an interest in modern information technology [17]. Data storage and protection are key aspects to ensure patients' acceptance of the procedure, particularly in European countries [34]. As adherence to digital care is broadly achieved by personal (patient-physician) interaction, we suggest that patients are anonymously categorized into different 'responder types' to streamline the processing of information. This strategy is already used in data-processing algorithms in social media [35] and may help prevent 'alert fatigue' caused by numerous repetitive or inconsequential appeals to the physician [17]. The optimized signaling cascade and information management can be exploited to achieve a 'physician-initiative' environment to counteract side effects or symptoms with a low-impact level on the patients' well-being. Eventually, financial and time expenditures in the general clinical management as well as for documentation might be reduced, allowing more time to be dedicated to direct patient interaction [36]. Figure 3 outlines the various aspirations, connected duties and concerns about electronic (e)PRO deployment for patients and physicians in clinical routine. Providing for 'analog lifelines' to foster the patients' determination to undergo therapy and participate in (e)PRO data collection was found to be a major aspect in the success of (e)PRO systems [17,33].

As the general public is increasing their online presence as well as becoming confident in data security [19,34], the opportunity for the scientific introduction of (e)PRO systems has arisen. It should also be noted that the implantation of digital care highly depends on the healthcare community's constant will to continuous improvements and - if necessary - fundamental changes. Prospective studies will have to transparently prove the clinical benefit of patient-reported outcomes throughout and following treatment. If proven clinically beneficial, these apps may even pave the way for novel approaches regarding the financial compensation to the health care provider, as PRO data sets may provide reliable information on actual treatment success and quality [37]. Nevertheless, the bioinformatic designs of such (e)PRO systems are constantly evolving and should consequently take a pivotal position in the ethical evaluation and monetary compensation of a certain product [35]. Ultimately, professional associations such as the ESTRO and ASTRO will have to be scientifically convinced in order to propagate and facilitate the implementation of (e)PRO-aided care into clinical routine.

\section{References}

1. Maloney DG, Grillo-López AJ, White CA, Bodkin D, Schilder RJ, Neidhart JA, et al. IDEC-C2B8 (rituximab) anti-CD20 monoclonal antibody therapy in patients with relapsed low-grade non-Hodgkin's lymphoma. Blood. 1997;

2. Trapani JA, Darcy PK. Immunotherapy of cancer. Aust Fam Physician. 2017;

3. Horn L, Mansfield AS, Szczęsna A, Havel L, Krzakowski M, Hochmair MJ, et al. First-Line Atezolizumab plus Chemotherapy in Extensive-Stage Small-Cell Lung Cancer. N Engl J Med. 2018;

4. Antonia SJ, Villegas A, Daniel D, Vicente D, Murakami S, Hui R, et al Durvalumab after Chemoradiotherapy in Stage III Non-Small-Cell Lung Cancer. N Engl J Med. 2017;

5. Thompson RF, Valdes G, Fuller CD, Carpenter CM, Morin O, Aneja S, et al. The Future of Artificial Intelligence in Radiation Oncology. International Journal of Radiation Oncology Biology Physics. 2018.

6. Tseng HH, Wei L, Cui S, Luo Y, Ten Haken RK, El Naqa I. Machine Learning and Imaging Informatics in Oncology. Oncology (Switzerland). 2020.
7. Izmajłowicz B, Rusiecka M, Sztuder A, Stępień M, Ignatowicz-Pacyna A, Słocka-Romaniuk B, et al. Tolerance of combined radiochemotherapy in cervical cancer patients. Adv Clin Exp Med. 2017;

8. Tanderup K, Nesvacil N, Kirchheiner K, Serban M, Spampinato S, Jensen NBK, et al. Evidence-Based Dose Planning Aims and Dose Prescription in ImageGuided Brachytherapy Combined With Radiochemotherapy in Locally Advanced Cervical Cancer. Seminars in Radiation Oncology. 2020.

9. Thomsen M, Vitetta L. Adjunctive Treatments for the Prevention of Chemotherapy- and RadiotherapyInduced Mucositis. Integrative Cancer Therapies. 2018.

10. Stewart MA. Effective physician-patient communication and health outcomes: A review. CMAJ. 1995.

11. Giordano FA, Welzel G, Siefert V, Jahnke L, Ganslandt T, Wenz F, et al. Digital Follow-Up and the Perspective of Patient-Centered Care in Oncology: What's the PROblem? Oncology (Switzerland). 2020.

12. Elkaddoum R, Haddad FG, Eid R, Kourie HR. Telemedicine for cancer patients during COVID-19 pandemic: Between threats and opportunities. Future Oncology. 2020.

13. Yildiz F, Oksuzoglu B. Teleoncology or telemedicine for oncology patients during the COVID-19 pandemic: the new normal for breast cancer survivors? Futur Oncol. 2020;

14. Cox A, Lucas G, Marcu A, Piano M, Grosvenor W, Mold F, et al. Cancer survivors' experience with telehealth: A systematic review and thematic synthesis. Journal of Medical Internet Research. 2017.

15. Van Tol-Geerdink JJ, Leer JWH, Van Oort IM, Van Lin EJNT, Weijerman PC, Vergunst H, et al. Quality of life after prostate cancer treatments in patients comparable at baseline. $\mathrm{Br} \mathrm{J}$ Cancer. 2013;

16. Mentz RJ, Hernandez AF, Berdan LG, Rorick T, O'Brien EC, Ibarra JC, et al. Good Clinical Practice Guidance and Pragmatic Clinical Trials : Balancing the Best of Both Worlds. Circulation. 2016;

17. Aiyegbusi OL, Kyte D, Cockwell P, Marshall T, Dutton M, Walmsley-Allen $\mathrm{N}$, et al. Patient and Clinician Perspectives on Electronic PatientReported Outcome Measures in the 
Management of Advanced CKD: A Qualitative Study. Am J Kidney Dis. 2019;

18. Banerjee A, Jadhav S, Bhawalkar J. Probability, clinical decision making and hypothesis testing. Ind Psychiatry J. 2009;

19. Statista. Internet usage in the USA [Internet]. 2020 [cited 2020 Sep 22]. Available

from: https://www.statista.com/topics/2237/int ernet-usage-in-the-united-states/

20. Partl R, Lehner J, Winkler P, Kapp KS. Testing the feasibility of augmented digital skin imaging to objectively compare the efficacy of topical treatments for radiodermatitis. PLoS One. 2019;

21. Chhabra HS, Sharma S, Verma S. Smartphone app in self-management of chronic low back pain: a randomized controlled trial. Eur Spine J. 2018;

22. Basch E, Barbera L, Kerrigan CL, Velikova G. Implementation of PatientReported Outcomes in Routine Medical Care. Am Soc Clin Oncol Educ B. 2018

23. Sloan JA, Cheville AL, Liu H, Novotny PJ, Wampfler JA, Garces YI, et al. Impact of self-reported physical activity and health promotion behaviors on lung cancer survivorship. Health Qual Life Outcomes. 2016;

24. Stijns RCH, De Graaf EJR, Punt CJA, Nagtegaal ID, Nuyttens JJME, Van Meerten E, et al. Long-term Oncological and Functional Outcomes of Chemoradiotherapy Followed by Organ-
Sparing Transanal Endoscopic Microsurgery for Distal Rectal Cancer: The CARTS Study. JAMA Surg. 2019;

25. Okumura Y, Inomata T, Iwata N, Sung J, Fujimoto K, Fujio K, et al. A review of dry eye questionnaires: Measuring patient-reported outcomes and healthrelated quality of life. Diagnostics. 2020.

26. Heydenreich M, Puta C, Gabriel HHW, Dietze A, Wright P, Zermann DH. Does trunk muscle training with an oscillating rod improve urinary incontinence after radical prostatectomy? A prospective randomized controlled trial. Clin Rehabil. 2020;

27. Donovan JL, Hamdy FC, Lane JA, Mason M, Metcalfe C, Walsh E, et al. Patient-reported outcomes after monitoring, surgery, or radiotherapy for prostate cancer. N Engl J Med. 2016;

28. Gagnier JJ. Patient reported outcomes in orthopaedics. Journal of Orthopaedic Research. 2017.

29. Tevis SE, James TA, Kuerer HM, Pusic AL, Yao KA, Merlino J, et al. PatientReported Outcomes for Breast Cancer. Ann Surg Oncol. 2018;

30. Verma V, Simone CB, Mishra M V. Quality of Life and Patient-Reported Outcomes Following Proton Radiation Therapy: A Systematic Review. Journal of the National Cancer Institute. 2018.

31. Widmark A, Gunnlaugsson A, Beckman L, Thellenberg-Karlsson C, Hoyer M, Lagerlund $\mathrm{M}$, et al. Ultrahypofractionated versus conventionally fractionated radiotherapy for prostate cancer: 5-year outcomes of the HYPORT-PC randomised, non-inferiority, phase 3 trial. Lancet. 2019;

32. Kirsh E, Naunheim M, Holman A, Kammer R, Varvares M, Goldsmith T. Patient-reported versus physiologic swallowing outcomes in patients with head and neck cancer after chemoradiation. Laryngoscope. 2019;

33. Wilkie JR, Mierzwa ML, Yao J, Eisbruch A, Feng M, Weyburne G, et al. Big data analysis of associations between patient reported outcomes, observer reported toxicities, and overall quality of life in head and neck cancer patients treated with radiation therapy. Radiother Oncol. 2019;

34. Statista. Perception of online data security in Germany 2014-2019 [Internet]. 2020 [cited 2020 Sep 22]. Available from: https://www.statista.com/statistics/4484 31/perception-of-online-data-securityin-germany/

35. Times NY. Liberal, Moderate or Conservative? See How Facebook Labels You [Internet]. 2020 [cited 2020 Sep 23]. Available from: https://www.nytimes.com/2016/08/24/u s/politics/facebook-ads-politics.html

36. Ammenwerth E, Spötl H-P. The Time Needed for Clinical Documentation versus Direct Patient Care. Methods Inf Med. 2009;

37. Porter ME. A Strategy for Health Care Reform - Toward a Value-Based System. N Engl J Med. 2009; 